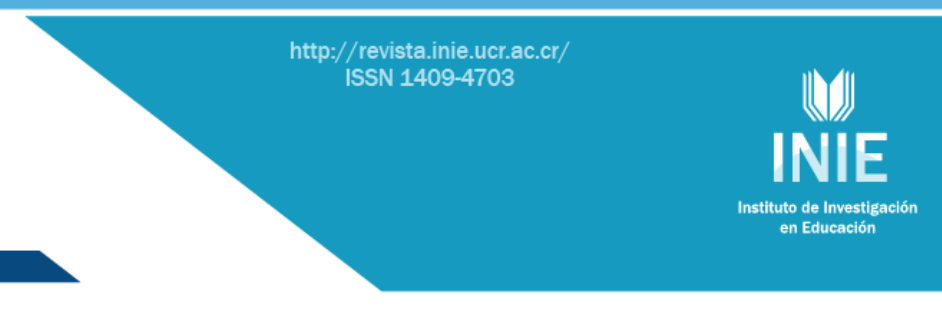

\title{
MOTIVACIÓN Y ESTRATEGIAS DE APRENDIZAJE DEL ESTUDIANTADO DE LA ESCUELA DE ORIENTACIÓN Y EDUCACIÓN ESPECIAL \\ MOTIVATION AND LEARNING STRATEGIES OF COUNSELING AND SPECIAL EDUCATION STUDENTS
}

\section{Volumen 14, Número 1}

Enero - Abril

pp. 1-20

Este número se publicó el 30 de enero de 2014

Sonia Parrales Rodríguez

Julieta Solórzano Salas

Revista indizada en REDALYC, $\underline{\text { SCIELO }}$

Revista distribuida en las bases de datos:

CATÁLOGO DE LATINDEX, IRESIE, CLASE, DIALNET, DOAJ, E-REVIST@S, SHERPA/ROMEO, QUALIS, MIAR

Revista registrada en los directorios:

ULRICH'S, $\underline{\text { REDIE}}, \underline{\text { RINACE}}, \underline{\text { OEI }}, \underline{\text { MAESTROTECA, PREAL, }}$ 


\title{
MOTIVACIÓN Y ESTRATEGIAS DE APRENDIZAJE DEL ESTUDIANTADO DE LA ESCUELA DE ORIENTACIÓN Y EDUCACIÓN ESPECIAL

\author{
MOTIVATION AND LEARNING STRATEGIES OF COUNSELING AND SPECIAL
} EDUCATION STUDENTS
}

\author{
Sonia Parrales Rodríguez \\ Julieta Solórzano Salas
}

\begin{abstract}
Resumen: El presente artículo científico expone los resultados de una investigación realizada en el año 2012 para caracterizar las estrategias de aprendizaje y la motivación de la población estudiantil de la Escuela de Orientación y Educación Especial, Facultad de Educación, Universidad de Costa Rica. Ambas carreras tienen un compromiso con la calidad y requieren contar con información que permita un mayor acercamiento a las formas en que cada estudiante se organiza para aprender, así como los aspectos que contribuyen a alcanzar el logro académico. En la investigación participó el estudiantado del Bachillerato en Ciencias de la Educación con énfasis en Educación Especial y del Bachillerato y la Licenciatura en Ciencias de la Educación con énfasis en Orientación. Se utilizó una adaptación de los instrumentos Cuestionario de Técnicas de estudio. Se seleccionaron los ítems que presentaron propiedades psicométricas para un total de 25 ítems con un Alfa de Cronbach de $a=0.785$. Como parte de los hallazgos de la investigación se obtiene que las estrategias de estudio que utiliza el grupo de estudiantes son: el repaso, el uso de esquemas, de los mapas conceptuales y del subrayado; pensamiento crítico, la metacognición, la autorregulación y el manejo de recursos. En el aspecto de motivación el estudiantado recurre a la motivación extrínseca e intrínseca y siente poca ansiedad ante los exámenes.
\end{abstract}

Palabras clave: ESTRATEGIAS DE APRENDIZAJE, MOTIVACIÓN, ESTUDIANTE UNIVERSITARIO, EDUCACIÓN SUPERIOR, COSTA RICA

\begin{abstract}
This scientific article presents the results of an investigation conducted in 2012 to characterize learning strategies and motivation of the student population of Counseling and Special Education, Faculty of Education, University of Costa Rica. Both careers have a commitment to quality and require information that allows a better approach to the ways in which each student organizes for learning, as well as the aspects that contribute to reach academic achievement. The research involved students of the Bachelor of Science in Education with an emphasis in Special Education and the Bachelor and Degree of Science in Education with emphasis in Counseling. We used an adaptation of two instruments: the Study Techniques Questionnaire and Motivated Strategies for Learning, and selected the items that showed psychometric properties for a total of 25 items with Cronbach's alpha of $\alpha=0.785$. As part of the research findings it is observed that the strategies of study used by the group of students were the review, the use of diagrams and concept maps, underlining, critical thinking, metacognition, self-regulation and resource management. In the motivation aspect, the student searches for intrinsic and extrinsic motivation and feels less anxiety on the exams.
\end{abstract}

Key words: LEARNING STRATEGIES, MOTIVATION, COLLEGE STUDENT, HIGHER EDUCATION, COSTA RICA

\footnotetext{
${ }_{1}$ Profesora de la Escuela de Orientación y Educación Especial, Universidad de Costa Rica. Licenciada en Educación con Énfasis en Orientación, Bachiller en Estadística. Dirección electrónica: sonia.parrales@ucr.ac.cr

2 Profesora de la Escuela de Orientación y Educación Especial, Universidad de Costa Rica. Investigadora del Instituto de Investigación en Educación (INIE). Máster en Evaluación Educativa.Dirección electrónica: julieta.solorzano@ucr.ac.cr
}

Artículo recibido: $1^{\circ}$ de julio, 2013

Devuelto para corrección: 3 de octubre, 2013

Aprobado: 12 de diciembre, 2013 


\section{Introducción}

La Escuela de Orientación y Educación Especial de la Facultad de Educación en Universidad de Costa Rica, forma profesionales en Orientación y en Educación Especial. Además, contribuye en la formación de educadores de preescolar, primaria y secundaria. A continuación se ofrece una sinopsis histórica de cada carrera.

La carrera de Orientación inició en 1964, y es pionera en la formación de profesionales en esta disciplina. Actualmente, se ofertan todos los años los grados académicos de Bachillerato y Licenciatura. La Orientación procura el

(...) autoconocimiento e identidad, conocimiento del contexto natural y sociocultural, la toma de decisiones y el planeamiento para la vida, con la finalidad de ayudar a las personas a clarificar y construir el sentido de vida que asumen para su existencia en las diferentes etapas y situaciones vitales. (Sección de Orientación, 2011, p. 64)

Por su parte, la carrera de Educación Especial inicia sus funciones en 1974. Actualmente, se oferta cada año el grado de Bachillerato. El programa de la Licenciatura se ofrece a la comunidad estudiantil cada dos años. Esta carrera ofrece al sistema educativo formal y no formal los apoyos para responder a las necesidades educativas especiales que presenta el estudiantado (Sección de Educación Especial, 2011).

A partir del año 2008, ambas carreras, con el apoyo del Centro de Evaluación Académica (CEA), se abocaron a la autoevaluación con fines de acreditación ante el Sistema Nacional de Acreditación de la Educación Superior (SINAES). Este proceso permitió a las carreras repensar las acciones cotidianas y su impacto social, cultural y educativo, debido a la naturaleza de cada objeto de estudio que las caracteriza. Producto del ejercicio de autovaloración, se identificaron áreas por mejorar, lo cual se plasmó en un compromiso de mejoramiento.

En el contexto de este artículo es importante rescatar que las Políticas de la Universidad de Costa Rica para los años 2010-2014 (Consejo Universitario, 2012) estimulan las tareas de autoevaluación y acreditación de las carreras, invitan a la flexibilización curricular, para así garantizar el óptimo cumplimiento de cada plan de estudios por parte del estudiantado y promover la innovación para liderar las transformaciones sociales que se requieran con el fin de garantizar la equidad y la justicia. 
Por el interés de mejoramiento e innovación, valorado desde el marco jurídico de la Universidad, ambas carreras han establecido en sus compromisos de mejora la consecución de un sistema de información de sus estudiantes (Sección de Educación Especial, 2011; Sección de Orientación, 2011), puesto que hay una necesidad palpable por acercarse a conocer las características demográficas y académicas que presenta el estudiantado, que es una de las poblaciones beneficiadas de la autoevaluación y la acreditación.

La formación profesional que pretende consolidar cada estudiante, desde una elección vocacional consciente, debe permitirle la construcción de los saberes conceptuales, procedimentales y actitudinales que contempla cada plan de estudios en el que se matriculó. La Universidad, como institución estatal de estudios superiores, tiene la responsabilidad social de velar por el cumplimiento y logro a cabalidad de la propuesta curricular planteada por cada carrera, puesto que es la Institución que certifica al estudiantado ante la sociedad.

Una forma de promover una adecuada formación en la educación superior, es demandar en el profesorado el conocimiento de las características de aprendizaje del estudiantado, de modo tal que la acción docente atienda esa caracterización y medie las estrategias de enseñanza y aprendizaje para el logro del perfil establecido en el plan de estudios acreditado. Méndez y González (2011, p. 6) indican que “(...) toda estrategia docente que se idee debe partir del conocimiento de cómo aprende el sujeto y cuáles son las variables que se deben manipular en el contexto educativo para favorecer ese aprendizaje (...)".

Por otro lado, el profesorado debe considerar la motivación que tiene la persona en formación con el fin de estimular no solamente el logro de la recompensa externa como aprobar el curso, sino también la valoración social y el incremento de la capacidad y profundidad del conocimiento. Arguedas (2010) afirma que "aunque hay variables relacionadas con la motivación que no se pueden cambiar, se puede determinar cuáles están sujetas a modificaciones que influyan para que el estudiantado mantenga o desarrolle su interés por aprender" (p. 31).

Esta investigación respondió a las siguientes interrogantes: ¿cuáles son las estrategias de aprendizaje que utiliza el estudiantado de las carreras de Educación Especial y Orientación?; ¿qué tipo de motivación para el estudio tiene este grupo de estudiantes? 


\section{Estrategias de aprendizaje y motivación}

Para dar respuesta a las cuestiones planteadas, se utilizaron los aportes de Pintrich y García (1993), Pintrich, Smith, García y McKeachie (1991, citados por Herrera y Lorenzo, 2009; Herrera, Jiménez y Castro, 2011), quienes señalan que en el estudiantado se pueden identificar tres tipos de estrategias de aprendizaje: las cognitivas (repaso, elaboración y organización de la información, además del pensamiento crítico); las metacognitivas (relacionadas con planificación, control y regulación de las actividades que la persona realiza durante el aprendizaje); y las de regulación de recursos (la organización del tiempo asignado y del ambiente de estudio, el esfuerzo aplicado, el aprendizaje con pares y la búsqueda de ayuda).

Por otra parte, se mencionan como estrategias de aprendizaje las siguientes: repaso, elaboración, organización, pensamiento crítico, metacognición y autorregulación, manejo de recursos (tiempo y ambiente), regulación del esfuerzo, aprendizaje con pares y búsqueda de ayuda, según Artino (2005). La investigación realizada utilizó el aporte teórico señalado para conceptualizar las estrategias de aprendizaje y de motivación del estudiantado.

Se entiende por repaso la actividad que lleva a la persona a repetir información para sí misma, es decir, repasar los materiales una y otra vez para incrementar la capacidad de evocación de información (Credé y Philips, 2011; Rotgans y Schmidt, 2010). Rinaudo, Chiecher y Donolo (2003) aclaran que esta estrategia promueve un procesamiento superficial de la información pues "(...) no ayudarían a construir conexiones internas o a integrar la nueva información con el conocimiento previo (...)" (p.109).

La elaboración son todas aquellas estrategias cognitivas para sintetizar e integrar información, tales como la toma de apuntes o el parafraseo (Zhi Feng y Hung, 2010; Rotgans y Schmidt, 2010).

Por su parte, la organización permite identificar las ideas principales o conceptos claves de una información y las ordena para lograr la resolución de problemas (Soprano, 2003). También es definida como la habilidad para manejar las demandas actuales o futuras de una tarea (Cooper-Kahn y Dietzel, 2008), que promueven procesamientos cognitivos más profundos de la materia a estudiar (Rinaudo, Chiecher y Donolo, 2003).

El pensamiento crítico se comprende como la habilidad que tiene cada estudiante para aplicar conocimientos previos a nuevos contextos, o evaluar críticamente ideas y conceptos. 
Esta habilidad permite desarrollar juicios reflexivos y realizar análisis de información según el contexto (Zhi Feng y Hung, 2010).

La metacognición y autorregulación permiten al estudiantado monitorear su propio desempeño y compararlo con los estándares de lo que se precisa o a partir de las metas autoestablecidas. Además, le posibilitan reflexionar sobre la efectividad de sus logros de aprendizaje (Cooper-Kahn y Dietzel, 2008; Credé y Philips, 2011).

El manejo de recursos son todas aquellas actividades que realiza el estudiante para calendarizar, planificar y administrar el propio tiempo de estudio y establecer metas realistas para el logro de los objetivos propuestos (Rotgans y Schmidt, 2010). El ambiente es considerado parte de los recursos de que se dispone, y se recomienda que sea tranquilo, ordenado y relativamente libre de distractores visuales o auditivos (Pintrich et al., 1991, citados por Rinaudo, Chiecher y Donolo, 2003).

La regulación del esfuerzo es la perseverancia que muestra la persona en el estudio aun cuando el material sea aburrido o difícil y demande un gran esfuerzo (Rotgans y Schmidt, 2010).

El aprendizaje con pares es la capacidad para estudiar colaborativamente con otras personas y realizar grupos para este fin (Rotgans y Schmidt 2010), mientras que buscar ayuda se da cuando el estudiantado muestra la habilidad de solicitar la asistencia o ayuda a compañeros de clase o docentes cuando se enfrenta a dificultades en el proceso de aprendizaje (Rotgans y Schmidt, 2010; Credé y Philips, 2011).

Las estrategias señaladas toman en cuenta la diversidad de formas de organización del trabajo individual y grupal para el logro de los aprendizajes universitarios.

Otro tema de interés en esta investigación fue la motivación para el estudio. Para comprender este tema se debe explorar la motivación intrínseca, la extrínseca, el valor de la tarea, el control de las creencias hacia el aprendizaje, las creencias de la autoeficacia para el aprendizaje y el rendimiento, así como la ansiedad (Pintrich, Smith, García, y McKeachie, 1991, citados por Artino, 2005). A continuación, se explican cada una de estas.

La motivación intrínseca le permite al estudiantado asumir una tarea de aprendizaje con deleite e interés (Rotgans y Schmidt, 2010; Hong, Greene y Hartzell, 2011). Es probable que una o un estudiante motivado intrínsecamente realice actividades que le signifiquen un desafío cognitivo o esté dispuesto a desarrollar tareas de aprendizaje que le exijan un pensamiento más profundo (Rinaudo, Chiecher y Donolo, 2003). Si las personas 
intrínsecamente motivadas cometen un error, deducen que es una forma de conocer en la que no está haciendo algo bien (Arguedas, 2010).

La motivación extrínseca es aquella que experimenta la persona cuando se compromete con una tarea de aprendizaje para buscar reconocimiento y recompensa (Hong, Greene y Hartzell, 2011; Rotgans y Schmidt, 2010). Las personas con este tipo de motivación realizan su trabajo por presión y por no reprobar, produciendo un aprendizaje menos creativo (Arguedas, 2010).

El valor de la tarea es uno de los dos componentes clave necesarios para comprender las conductas de logro y los resultados académicos de cada estudiante; se refiere al grado en que consideran que una tarea académica tiene valor o vale la pena ser realizada (Liem, Lau y Nie, 2007). Los juicios que realiza el grupo de estudiantes sobre qué tan interesante, útil e importante es una tarea, condicionan el grado de involucramiento que va a asumir (Rotgans y Schmidt, 2010).

El control de las creencias hacia el aprendizaje corresponde a las creencias del estudiantado de que los resultados devienen de su propio esfuerzo y no a factores externos como el docente, por lo que, a mayor empeño, es mayor la posibilidad de obtener resultados positivos (Zhi Feng y Hung, 2010; Rotgans y Schmidt, 2010).

Las creencias de la autoeficacia para el aprendizaje y el rendimiento llevan al estudiantado a tener confianza de realizar las tareas que se le asignan (Credé y Philips, 2011). La autoeficacia incorpora la expectativa del éxito, es específica del desempeño de la tarea y de los juicios sobre la propia habilidad de completar una tarea y confiar en las propias habilidades para realizarla (Rotgans y Schmidt, 2010).

Por último, la ansiedad es la emoción negativa o la preocupación que genera la realización de pruebas y exámenes (Zhi Feng y Hung, 2010).

Las teorías sobre la motivación tienen un gran valor para el profesorado en la educación superior, porque facilitan el entendimiento de las conductas hacia las tareas, así como del rendimiento.

\section{Método}

Se trata de una investigación cuantitativa de tipo descriptivo. Según Hernández, Fernández y Baptista (2010) este enfoque recolecta datos para realizar análisis estadístico y determinar formas de comportamiento en el grupo examinado. Además, es de tipo 
descriptivo pues mide las variables de estudio y las describe sin dar una explicación de la forma como se relacionan. Más bien, contribuye a tener un panorama más amplio y preciso de las estrategias de aprendizaje y la motivación del grupo de estudiantes que participaron.

\section{Participantes y contexto de aplicación}

Participó el estudiantado de las carreras del Bachillerato en Ciencias de la Educación con énfasis en Educación Especial y de la Licenciatura y Bachillerato en Ciencias de la Educación con énfasis en Orientación presentes en los cursos del segundo ciclo del 2012 en el momento de la aplicación. La participación fue voluntaria y se visitó un curso por nivel para un total de 80 estudiantes de Educación Especial y 100 de Orientación (Tabla 1).

Tabla 1: Participación por curso en cada carrera

\begin{tabular}{|c|c|c|}
\hline \multirow[b]{2}{*}{ Curso } & \multicolumn{2}{|c|}{ Carrera } \\
\hline & $\begin{array}{c}\text { Educación } \\
\text { Especial }\end{array}$ & Orientación \\
\hline OE0205 - Estimulación temprana para el desarrollo integral & 27 & \\
\hline OE0184 - Evaluación para el planeamiento curricular & 10 & \\
\hline OE0195 - Estrategias metodológicas específicas V & 19 & \\
\hline OE0172 - Práctica supervisada & 24 & \\
\hline OE2033 - Orientación e Identidad de género & & 18 \\
\hline FD0133 - Planeamiento educativo y curricular & & 12 \\
\hline OE2001 - Introducción a la orientación & & 30 \\
\hline OE2058 - Orientación vocacional-ocupacional II & & 12 \\
\hline $\begin{array}{l}\text { OE2057 - Orientación a Personas en Situaciones de Riesgo } \\
\text { Psicosocial }\end{array}$ & & 11 \\
\hline OE2056 - Orientación en el entorno educativo II & & 4 \\
\hline OE2045 - Práctica Profesional II & & 13 \\
\hline Total & 80 & 100 \\
\hline
\end{tabular}

Fuente: Construcción propia, 2013.

\section{Instrumentos}

La aplicación de instrumentos se realizó en la primera semana del mes de diciembre del año 2012.

Debido a los objetivos planteados, se utilizaron los cuestionarios de Técnicas de estudio y el Motivated Strategies for Learning, que han evidenciado confiabilidad estadística en otras investigaciones, la cual se expondrá a continuación. 
El Cuestionario de Técnicas de estudio fue diseñado y aplicado por Herrera y Lorenzo (2009) y Herrera, Jiménez y Castro (2011) con estudiantes de primer y último año de psicología y docencia en cuatro universidades españolas. Este instrumento tiene una confiabilidad interna de $\alpha=0.72$ y está integrado por cuatro secciones que abordan los siguientes aspectos:

I sección: Datos de identificación de las personas participantes, tales como sexo y edad. Además, pregunta por la titulación y especialidad, curso y vía de acceso a la universidad. Sin embargo, estos aspectos no se consideraron pertinentes para la realidad del estudiantado de las carreras de Orientación y Educación Especial.

II sección: Son ítems que permiten conocer el lugar y las condiciones de estudio del grupo de estudiantes. Estos ítems obtuvieron un $\alpha=0.65$.

III sección: Con un $\alpha=0.67$, se exploran las estrategias que cada estudiante utiliza para organizar el estudio, dentro de las cuales se incluye planificación del tiempo de estudio y la información que utiliza para establecer prioridades a la hora de estudiar.

IV sección: Interesa conocer la frecuencia de algunas estrategias que se dan antes, durante y después del estudio. Esta sección presentó un $\alpha=0.82$.

Por su parte, el cuestionario Motivated Strategies for Learning (MSLQ) de Pintrich, Smith, García, y McKeachie (1991, citado por Artino 2005) aportó reactivos para medir la motivación del estudiantado en el aprendizaje. Según explica Artino (2005), el MSLQ está compuesto por 81 ítems, agrupados en dos categorías:

1. La motivación: se compone de 31 ítems. EI MSLQ valora las metas y las creencias que tiene cada estudiante de las habilidades para enfrentar un curso y la ansiedad ante las pruebas. Según la tabla 2 , este conjunto de reactivos se agrupan en las siguientes subescalas y sus respectivos valores del coeficiente alfa.

Tabla 2: Subescalas del cuestionario MSLQ

\begin{tabular}{|l|c|c|}
\hline \multicolumn{1}{|c|}{ Sub escala } & \multicolumn{1}{c|}{$\begin{array}{c}\text { Cantidad } \\
\text { de ítems }\end{array}$} & $\boldsymbol{\alpha}$ \\
\hline Motivación intrínseca & 4 & $\mathbf{0 . 7 4}$ \\
\hline Motivación extrínseca & 4 & $\mathbf{0 . 6 2}$ \\
\hline El valor de la tarea & 6 & $\mathbf{0 . 9 0}$ \\
\hline Control de las creencias hacia el aprendizaje & 4 & $\mathbf{0 . 6 8}$ \\
\hline $\begin{array}{l}\text { Creencias de la autoeficacia para el aprendizaje y el } \\
\text { rendimiento }\end{array}$ & 8 & $\mathbf{0 . 9 3}$ \\
\hline Ansiedad & $\mathbf{5}$ & $\mathbf{0 . 8 0}$ \\
\hline
\end{tabular}

Fuente: Artino (2005, p. 20) 
2. Las estrategias de aprendizaje: explora, con 50 ítems, las estrategias cognitivas y metacognitivas de uso frecuente para el estudiantado y se encuentran agrupados en nueve subescalas tal y como se muestra en la tabla 3.

Tabla 3: Subescalas del cuestionario Cuestionario Técnicas de estudio

\begin{tabular}{|l|c|c|}
\hline \multicolumn{1}{c|}{ Sub escala } & Cantidad de ítems & $\boldsymbol{\alpha}$ \\
\hline Repaso & 4 & $\mathbf{0 . 6 9}$ \\
\hline Elaboración & 6 & $\mathbf{0 . 7 5}$ \\
\hline Organización & 4 & $\mathbf{0 . 6 4}$ \\
\hline Pensamiento crítico & 5 & $\mathbf{0 . 8 0}$ \\
\hline Metacognición y autorregulación & 12 & $\mathbf{0 . 7 9}$ \\
\hline Manejo de recursos & 8 & $\mathbf{0 . 7 6}$ \\
\hline Regulación del esfuerzo & 4 & $\mathbf{0 . 6 9}$ \\
\hline Aprendizaje con pares & 3 & $\mathbf{0 . 7 6}$ \\
\hline Buscar ayuda & $\mathbf{4}$ & $\mathbf{0 . 5 2}$ \\
\hline
\end{tabular}

Fuente: Artino (2005, p. 20)

\section{Descripción del cuestionario elaborado para esta investigación}

Las investigadoras se dieron a la tarea de unir los cuestionarios Técnicas de Estudio y el MSLQ. Para esto se hizo una discusión de los diferentes ítems que contenían cada uno y las similitudes existentes. Es importante anotar que, debido a que el MSLQ enfoca el interés a la motivación aplicada a un curso puntualmente, se hizo la adaptación en la redacción para que el estudiantado valorara su motivación para la carrera y se eliminaron algunos ítems. El cuestionario quedó estructurado inicialmente con un total de 115 reactivos.

Para la validación de contenido se solicitó la evaluación de tres personas expertas con grado de maestría y experiencia en la docencia universitaria. Se seleccionaron los ítems que presentaron el acuerdo de al menos dos jueces, para un total de 59 reactivos en la versión final. Se les solicitó a las estudiantes del Bachillerato en Ciencias de la Educación con énfasis en Educación Especial de II y III nivel (Recinto de Guápiles, Universidad de Costa Rica) que hicieran una revisión del instrumento para corroborar la claridad y comprensión de los ítems.

- I parte de datos generales: para esta investigación se incluyeron las variables sociodemográficas de sexo, edad, estado civil, trabajo remunerado, jornada de trabajo. Además, se incorporaron otros aspectos para caracterizar la condición del grupo de 
estudiantes, tanto en el contexto de la Universidad como en la carrera tales como: tipo de opción en la elección de la carrera, si está cursando otra carrera; cantidad de cursos matriculados en la Universidad y en la carrera; y la valoración de la percepción del grado de dificultad de la carrera.

- Il parte: con 59 reactivos se pretendía medir las estrategias de estudio y la motivación para el aprendizaje.

\section{Análisis de los datos}

La información recopilada se procesó con el programa SPSS (versión 20). Para considerar las propiedades psicométricas de los reactivos se utilizó la correlación de cada ítem con la totalidad del instrumento. Se seleccionaron aquellos ítems que alcanzaron el estadístico $\leq 0.25$, para un total de 25 ítems con un Alfa de Cronbach de $\alpha=0.785$ para medir los siguientes indicadores: motivación (intrínseca, extrínseca y ansiedad) y estrategias de aprendizaje (repaso, elaboración, organización, pensamiento crítico, metacognición, manejo de recursos y buscar ayuda). Los ítems se pueden consultar en el anexo 1.

\section{Análisis de los resultados}

A continuación, se exponen los resultados obtenidos de la aplicación del cuestionario que permitieron identificar las estrategias de aprendizaje y el tipo de motivación del estudiantado de ambas carreras. Es necesario aclarar que en Orientación no se presentaron diferencias significativas entre las respuestas de los estudiantes del nivel de bachillerato con el de licenciatura.

\section{Características de la población informante}

Se puede observar en la Tabla 4 que, para ambas carreras, la mayor cantidad de estudiantes son mujeres, solteras, no trabajan o realizan jornadas de trabajo por horas. La edad promedio para Educación Especial es de 21 años y en la carrera de Orientación, 23.

En la siguiente tabla se resumen las características personales y académicas del estudiantado participante. 
Tabla 4: Características del estudiantado de las carreras de Educación Especial y Orientación consultado

\begin{tabular}{|l|c|c|c|c|}
\hline & \multicolumn{5}{|c|}{$\begin{array}{c}\text { Educación } \\
\text { Especial (N:80) }\end{array}$} & $\begin{array}{c}\text { Orientación } \\
(\mathrm{N}: 100)\end{array}$ \\
\hline & Abs. & $\%$ & Abs. & $\%$ \\
\hline Sexo & & & & \\
\hline Mujeres & 77 & $96 \%$ & 80 & $80 \%$ \\
\hline Hombres & 3 & $4 \%$ & 20 & $20 \%$ \\
\hline Estado civil & & & & \\
\hline Soltero (a) & 79 & $99 \%$ & 91 & $91 \%$ \\
\hline Casado (a) - unión libre & 1 & $1 \%$ & 8 & $8 \%$ \\
\hline Viudo (a) - divorciado (a) & - & - & 1 & $1 \%$ \\
\hline Trabajo remunerado & & & & \\
\hline No & 62 & $78 \%$ & 67 & $67 \%$ \\
\hline Sí & 18 & $22 \%$ & 32 & $32 \%$ \\
\hline Jornada de trabajo & & & & \\
\hline Por horas & 10 & $56 \%$ & 17 & $53 \%$ \\
\hline Medio tiempo & 5 & $28 \%$ & 7 & $22 \%$ \\
\hline Cuarto tiempo & 2 & $11 \%$ & 3 & $9 \%$ \\
\hline Tiempo completo & 1 & $6 \%$ & 5 & $16 \%$ \\
\hline Edad promedio & \multicolumn{3}{|c|}{20,82} & 22,68 \\
\hline Desviación estándar & \multicolumn{3}{|c|}{1,87} & \multicolumn{2}{c|}{5,26} \\
\hline
\end{tabular}

Fuente: Resultados de la encuesta realizada al grupo de estudiantes participantes (2012).

De acuerdo con las características académicas, se presenta en común que una cuarta parte afirma estar empadronado en otra carrera, de la cual poco más del $60 \%$ es del área de las Ciencias Sociales (47\% son de la Facultad de Educación para los estudiantes de Educación Especial y un 31\% en el caso del estudiantado en Orientación). Para el 36\% de Educación Especial, esta fue su primera opción de carrera al ingresar a la Universidad, mientras que en Orientación fue el 50\%.

Como otra característica académica, se observa que el grupo de estudiantes de Educación Especial matricula más cursos (cinco cursos, de los cuales cuatro corresponden a la carrera) y de manera consistente asigna más número de horas para estudiar $(\bar{x}=16$ horas semanales). Por su parte, el estudiantado de la carrera de Orientación matricula 4 cursos de la carrera y asigna $\bar{x}=12$ horas semanales para estudiar.

Dado lo anterior, es criterio de las investigadoras que los resultados obtenidos son pertinentes a la realidad de ambas carreras, en cuanto a que la mayoría del estudiantado son mujeres y en la carrera de Orientación se matriculan personas de más edad que el promedio. 
Con respecto a la valoración de la carrera, un $56 \%$ indica que tiene la misma dificultad que otras carreras de educación y un $62 \%$ indica que tiene el mismo grado de dificultad con respecto a otras áreas.

\section{Motivación}

Para el análisis de este apartado se incluyeron los indicadores de motivación intrínseca y extrínseca y ansiedad ante los exámenes.

Con respecto a la motivación intrínseca, los grupos de ambas carreras coinciden en que les resulta satisfactorio tratar de comprender el contenido que se presenta en los cursos, tan profundamente como sea posible (88\%). Un porcentaje menor (72\%) se refiere a que lo más importante es mejorar el promedio general, así que el objetivo a lograr en los cursos de la carrera es obtener una buena nota. De esta manera, se puede afirmar que el estudiantado es motivado por la nota, pero es más importante interiorizar y profundizar en los aprendizajes, por lo tanto, implican un desafío cognitivo y están dispuestos a desarrollar tareas de aprendizaje que exigen un pensamiento más profundo.

Del indicador motivación extrínseca, el $74 \%$ de estudiantes opinan que quieren hacer bien las cosas para demostrar sus capacidades a las personas que les son significativas. En este sentido, la formación profesional permite desarrollar y potencializar habilidades profesionales que pueden ser manifestadas en diferentes ámbitos como el familiar y el laboral, los cuales proveen el reconocimiento y recompensa que caracteriza este tipo de motivación.

Según lo anterior, es oportuno invitar al profesorado de ambas carreras a reflexionar si la entrega de la docencia es coincidente con los aspectos que motivan tanto intrínseca como extrínsecamente al estudiantado, considerando los espacios de aprendizaje como una oportunidad para la interiorización de conocimientos y el desarrollo de habilidades disciplinares.

Otro indicador que se exploró fue la ansiedad que genera en el estudiantado el examen, por lo que se indagó la frecuencia del uso de esta estrategia en ambas carreras y el nivel de agrado por parte de las personas informantes.

En el caso de Educación Especial se incluyen exámenes en el $75 \%$ de los cursos y en Orientación en el $50 \%$ de los cursos. Al consultarles el nivel de agrado por esta estrategia, es 
mayor el porcentaje de estudiantes de Educación Especial que se mostró indiferente (45\%), mientras que el estudiantado de Orientación tiende a la opción del desagrado (40\%).

Del grupo de estudiantes participantes de ambas carreras, un $21 \%$ reconoce que le agradan los exámenes como estrategia de evaluación, por lo que el 79\% restante podría sentirse poco motivado en los cursos que los incluyan. Al consultarles sobre sus reacciones, el $51 \%$ admite que siente nervios cuando realiza exámenes, pero no todos tienen manifestaciones físicas de ansiedad como el latido acelerado del corazón (26\%) y sensación de inquietud y malestar (38\%). Un 59\% piensa en las preguntas que no pudo responder al salir del examen y las corrobora con los apuntes que ha realizado en el curso (54\%); el 57\% analiza las posibles consecuencias ante una baja calificación. Contrario a lo que se podría esperar, el estudiantado presenta niveles aceptables de ansiedad ante los exámenes, es decir, no genera emociones negativas o preocupación; para la mitad del grupo, una vez concluida la prueba no hay preocupación por el resultado o por corroborar las respuestas.

\section{Técnicas de estudio}

El estudiantado de ambas carreras coincide en que prefiere estudiar todos los días de la semana. Sin embargo, el porcentaje de estudiantes de Educación Especial con una franja horaria de estudio definida (de $7 \mathrm{pm}$. a media noche) es mayor que el grupo de estudiantes de Orientación en este mismo horario, tal y como se detalla en el Gráfico 1. Es interesante observar que ambos grupos coinciden en los horarios en que inician el estudio, pero el grupo de Educación Especial concluye en horas más avanzadas de la noche; esto podría justificarse por el hecho de que, en promedio, matriculan más cursos y, por lo tanto, dedican más horas de estudio. 


\section{Gráfico 1: Franjas horarias de estudio del estudiantado de la carrera de Educación Especial y Orientación}

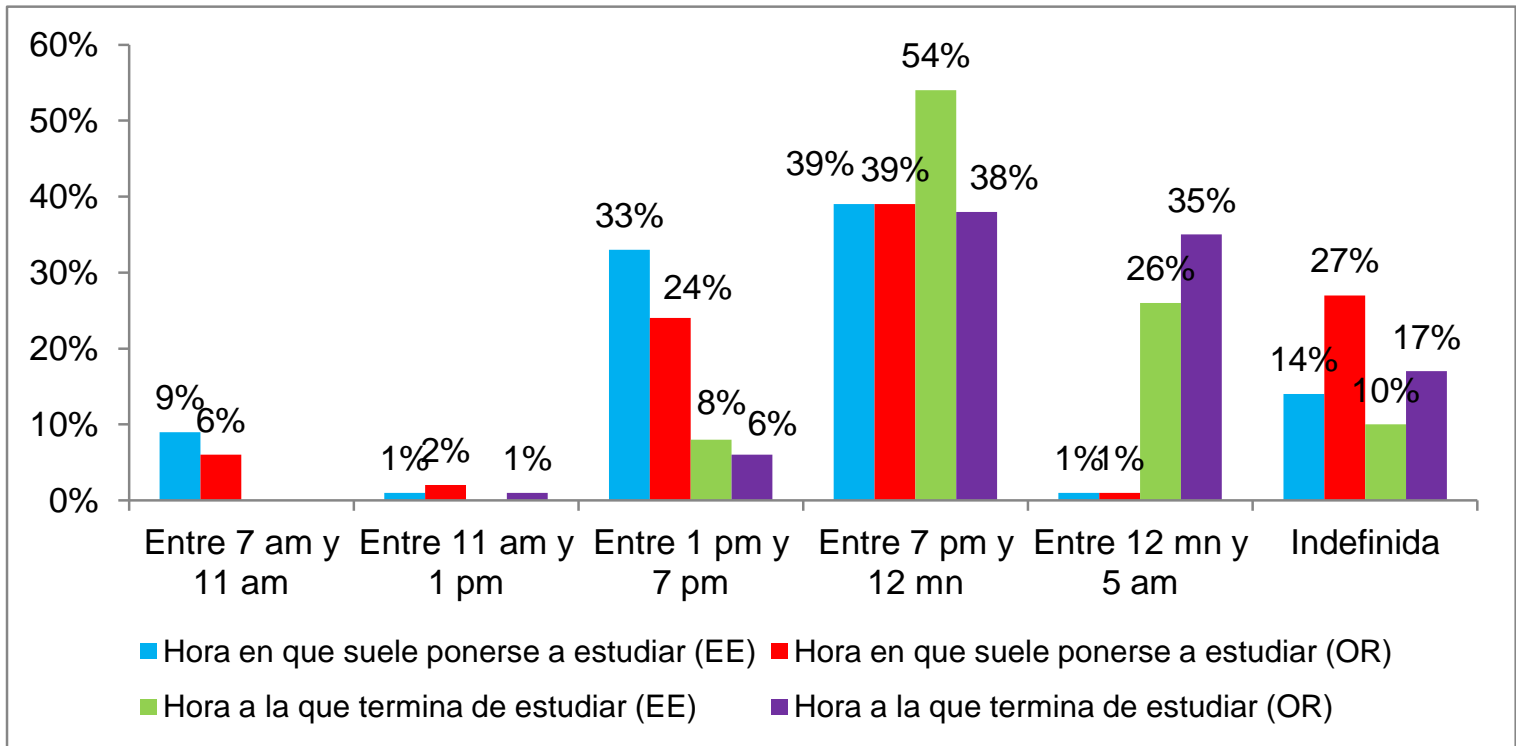

Fuente: Resultados de la encuesta realizada al grupo de estudiantes participantes (2012).

A continuación, se exponen los resultados de los indicadores: repaso, elaboración, organización, pensamiento crítico, metacognición y autorregulación, manejo de recursos y búsqueda de ayuda.

Para el indicador repaso, el grupo de examinados y examinadas indica que realiza una ojeada rápida a la materia antes de profundizar. Sin embargo, el estudiantado de Orientación repasa los apuntes más que el de Educación Especial (46\%, 40\% respectivamente, p <.05), pero los estudiantes de esta última carrera leen con mayor frecuencia sus comentarios anotados y repasan las lecturas en varias oportunidades $(62 \%, 22 \%$ respectivamente, $p<$ .05). La estrategia de repaso puede ser utilizada por el profesorado de ambas carreras para priorizar los conceptos y los contenidos que son importantes para la disciplina, teniendo en cuenta que promueve un procesamiento superficial de la información y facilitan la capacidad de evocación.

Como es evidente, en ambos grupos se observan diferencias en las técnicas de repaso, por lo tanto, el profesorado puede hacer uso de estas estrategias para mediar los aprendizajes en el aula, o bien incentivar el uso de aquellas otras que se utilizan de manera menos frecuente o no se conocen. 
Para el indicador elaboración, el estudiantado de ambas carreras considera que no es necesario el uso de muchas palabras en la elaboración de esquemas, los cuales son más usados que los mapas conceptuales. Sin embargo, el grupo de Educación Especial utiliza más esta técnica que los de Orientación $(49 \%, p<.05)$. Ambos grupos se dividen en igual porcentaje al utilizar o no utilizar frases de los apuntes, libros y otros materiales del curso para realizar los esquemas.

Cuando el estudiantado de ambas carreras se refiere al uso de esquemas y mapas conceptuales para estudiar, se puede afirmar que la elaboración que hacen de los contenidos desarrollados durante la clase, tiende a ser concreto, con pocas palabras y sistematizado. Este tipo de estrategias favorece la integración de los diferentes contenidos.

En cuanto al indicador organización, el 93\% del grupo de estudiantes participantes subraya las ideas principales en el material de estudio. Esto confirma el interés del estudiantado en rescatar lo esencial de los contenidos estudiados para procesar cognitivamente el material de estudio, por lo tanto, el profesorado puede recurrir a una diversidad de estrategias para mediar el aprendizaje; pero, a partir de los resultados de esta investigación, se sugiere que, además, se le ayude al estudiantado a establecer los conceptos claves.

Del indicador pensamiento crítico, se observa que el $80 \%$ de estudiantes indicó que procura aplicar las ideas de las lecturas en otras actividades de aprendizaje, así como las exposiciones y las discusiones en clase. Por lo tanto, el aprendizaje se construye como una experiencia integrada, transferible a otras prácticas de aprendizaje, evidenciando la existencia de temas transversales en la formación que les permite unificar los conocimientos y el desarrollo de juicios reflexivos.

En metacognición y autorregulación, se observa que la mitad del estudiantado emplea actividades tales como hacer preguntas que les ayudan a enfocar la lectura y hacer uso de material que les causa curiosidad, aunque se les dificulte entenderlo; además, el 60\% prefiere tener material que los rete para aprender nuevas cosas. Solamente el $42 \%$ admite que utiliza el diccionario para aclarar términos o palabras confusas. El profesorado debe aprovechar el interés que muestra el estudiantado por el desafío cognitivo para ayudarlos a establecer metas de aprendizaje alcanzables, mediadas por la autorregulación. En el indicador buscar ayuda, se confirma esta situación porque solamente el $54 \%$ del estudiantado reconoce que pregunta al profesor o profesora para clarificar los conceptos 
vagos. En este sentido, un ambiente en el aula en el que el estudiantado teme a equivocarse, puede ser una de las razones para sentir ansiedad y poca confianza para pedir ayuda cuando la necesita.

En cuanto al manejo de recursos, el $89 \%$ de estudiantes procura tener a mano la información y los materiales que requiere cada vez que estudia y un $84 \%$ cuenta con computadora en el lugar que tiene dispuesto para estudiar. Un porcentaje similar (87\%) confirma que cuenta con un grupo de pares para realizar los trabajos académicos y para estudiar. En relación con este indicador, el estudiantado cuenta con adecuadas técnicas y recursos para estudiar, lo cual se puede valorar como un factor a favor del desempeño académico.

\section{Conclusiones}

Como parte del compromiso de mejora y la búsqueda de la calidad que tienen ambas carreras, es necesario profundizar en las características demográficas y de aprendizaje del estudiantado para ofrecer una respuesta educativa acorde a este perfil.

En función de los objetivos propuestos en la investigación, se presentan las características en las estrategias de estudio y la motivación de la población consultada.

El estudiantado de Educación Especial y Orientación estudia todos los días de la semana y lo hace especialmente en la franja horaria de 7 p.m. a media noche. Los de Educación Especial realizan el repaso con una "ojeada" rápida a la materia, leen con mayor frecuencia los comentarios anotados y releen las lecturas en varias oportunidades antes de profundizar; en el caso de Orientación, revisan los apuntes. El grupo de docentes puede incentivar al estudiantado a controlar su trabajo elaborando un horario de estudio como estrategia adicional de motivación. Con respecto a las estrategias de estudio, se recomienda un aprendizaje en profundidad más que uno superficial. Este tipo de aprendizaje ayuda a relacionar la teoría con la vivencia, retomando la relevancia que tiene para la persona.

Para el indicador elaboración, el estudiantado de ambas carreras considera que no es necesario el uso de muchas palabras en la elaboración de esquemas, los cuales son más usados que los mapas conceptuales. En la organización, el subrayado de las ideas principales en el material de estudio es la estrategia más utilizada. Aunque esta técnica de asociación demanda poco tiempo y esfuerzo, no hay un entendimiento real de los conceptos. En cuanto al pensamiento crítico, tiende a aplicar las ideas de las lecturas en otras 
actividades de aprendizaje como las exposiciones y las discusiones en clase. En este sentido, el pensamiento reflexivo individual ayuda a apropiarse de los aprendizajes.

En metacognición y autorregulación, el grupo de estudiantes utiliza actividades como hacer preguntas que les ayudan a enfocar la lectura, hacer uso de material que les causa curiosidad, aunque se les dificulte entenderlo y prefiere tener material que los rete para aprender nuevas cosas. No siempre utilizan el diccionario para aclarar términos o palabras confusas. Al respecto, el grupo docente puede proveer estrategias didácticas que proporcionen las condiciones básicas para que los procesos metacognitivos se puedan dar, como, por ejemplo, estimular la exploración de los estilos de aprendizaje y el uso de recursos que están al alcance, como el diccionario.

En el manejo de recursos, la mayoría de estudiantes procura tener a mano la información y los materiales que requiere cada vez que estudia, y cuenta con computadora en el lugar que tiene dispuesto para estudiar. Además, se organizan en grupo para realizar los trabajos académicos y para estudiar. Un trabajo en grupo productivo proporciona la oportunidad para la ayuda mutua de la resolución de tareas, pero el profesorado también puede estimular el análisis de controversias conceptuales.

En relación con la motivación para el estudio, el grupo de estudiantes indica, con respecto a la motivación intrínseca, que les resulta satisfactorio tratar de comprender el contenido que se presenta en los cursos de la carrera, tan profundamente como sea posible, y lo más importante es mejorar el promedio general. En motivación extrínseca, valoran la posibilidad de demostrar sus capacidades a las personas que les son significativas, por lo tanto buscan el reconocimiento. El profesorado puede valorar el tipo de motivación que cada una de las estrategias de enseñanza y evaluación provoca en su grupo.

Ante la ansiedad que genera el examen en el estudiantado, pocos reconocen que les agradan los exámenes como evaluación, pero sólo la mitad manifiesta emociones negativas o preocupación. Aunque esta estrategia se asocia con motivación externa, es necesaria para la valoración de los aprendizajes y cada docente puede estimar si beneficia el propio desarrollo o si genera desagrado por miedo al fracaso.

El profesorado de ambas carreras puede utilizar la información que se desprende de esta investigación para mediar un aprendizaje significativo, con estrategias de uso frecuente, o bien implementar otras que se ajusten a las motivaciones del estudiantado. 
Ante los resultados obtenidos se recomienda al profesorado de la Escuela promover en el estudiantado las estrategias de aprendizaje que se señalan en el referente teórico, y que no son utilizadas por el estudiantado, tales como la regulación del esfuerzo y el aprendizaje con pares. Esta última genera, incluso, la interdependencia positiva entre el grupo de estudiantes.

Del mismo modo, con la motivación, el profesorado puede hacer uso de la valoración de las diferentes tareas que realizan para adquirir el aprendizaje, controlar las creencias hacia el aprendizaje, la propia eficiencia y el desempeño personal para el éxito académico.

\section{Referencias}

Arguedas, Irma. (2010). Promoción de la permanencia de estudiantes en la educación secundaria. Manual de temas y estrategias. San José: INIE.

Artino, Anthony. (2005). Review of the Motivated Strategies for Learning Questionnaire. Recuperado http://www.sp.uconn.edu/ aja05001/comps/documents/MSLQ Artino.pdf

Consejo Universitario, Universidad de Costa Rica. (2012). Políticas de la Universidad de Costa Rica para los años 2010-2014. Recuperado de: http://www.cu.ucr.ac.cr/normativ/politicas institucionales 2010-2014

Cooper-Kahn, Joyce y Dietzel, Laurie. (2008). Late, lost and unprepared. A paretn's guide to helping children with executive functioning. United States of America: Woodbine House.

Credé, Marcus y Philips, Alison. (2011). A meta-analytic review of the Motivated Strategies for Learning Questionarie. Learning and Individual Differences, 21, 337-346.

Hernández, Roberto, Fernández, Carlos y Baptista, Pilar. (2006). Metodología de la investigación (4ta ed.). México: Mc Graw Hill.

Herrera, Lucía y Lorenzo, Oswaldo. (2009). Estrategias de aprendizaje en estudiantes universitarios. Un aporte a la construcción del Espacio Europeo de Educación Superior. Educación y Educadores, 12(3), 75-98.

Herrera, Lucía, Jiménez, Gracia y Castro, Ángel. (2011). Aprendizajes del alumnado universitario de primer y último curso de las titulaciones de Psicología y Magisterio. Electronic Journal of Research in Educational Psychology, 9(2), 659-692.

Hong, Eunsook, Greene, Marie y Hartzell, Stephanie. (october, 2011). Cognitive and motivational characteristics of elementary teachers in general education classrooms and in gifted programs. Gifted child quarterly, 55(42), 50-264. 
Liem, Arief, Lau, Shun y Nie, Youyan. (2007). The role of self-efficacy, task value, and achievement goals in predicting learning strategies, task disengagement, peer relationship, and achievement outcome. Contemporary Educational Psychology, 33, 486-512.

Sección de Educación Especial, Escuela de Orientación y Educación Especial, Universidad de Costa Rica. (2011). Informe de autoevaluación de la carrera de Bachillerato en Ciencias de la Educación con énfasis en Educación Especial. San José: Escuela de Orientación y Educación Especial.

Sección de Orientación, Escuela de Orientación y Educación Especial, Universidad de Costa Rica. (2011). Informe de autoevaluación de la carrera Bachillerato y Licenciatura en Ciencias de la Educación con énfasis en Educación Especial. San José: Escuela de Orientación y Educación Especial.

Méndez, Luz y González, Mónica. (2011). Escala de estrategias docentes para aprendizajes significativos: diseño y evaluación de sus propiedades psicométricas. Revista Actualidades Investigativas en Educación, 11(3). Recuperado de http://revista.inie.ucr.ac.cr/ediciones/controlador/Article/accion/show/articulo/escala-deestrategias-docentes-para-aprendizajes-significativos-diseno-y-evaluacion-de-suspropiedades-psicometricas.html

Rinaudo, María Cristina, Chiecher, Analía y Donolo, Danilo. (2003). Motivación y uso de estrategias en estudiantes universitarios. Su evaluación a partir del Motivated Strategies Learning Questionnaire. Anales de Psicología, 19(1), 107-119.

Rotgans, Jerome y Schmidt, Henk. (2010). The Motivate Strategies for Learning Questionnaire: A meassure for student's general motivational beliefs and learning strategies?. The Assia-Pacific Education Researcher, 19(2), 357-369.

Soprano, Ana María. (2003). Evaluación de las funciones ejecutivas en el niño. Revista de Neurología, 37(1), 44-50.

Zhi Feng, Eric y Hung, Chung. (april, 2010). The survey study of mathematics motivated strategies for learning questionnaire (MMSLQ) for grade 10-12 Taiwanese Students. The Turkish Online Journal of Educational Technology, 9(2), 221-233. 


\section{Anexo 1. Ítems utilizados en el análisis de los resultados}

\section{Repaso}

Corrected

Cuando estudio para este curso, leo mis notas y las lecturas una y otra vez.

Repaso los apuntes frecuentemente

Correlation

Antes de estudiar en profundidad, primero realizo una lectura superficial de la materia

\section{Elaboración}

Hago esquemas de la materia que voy a estudiar

Hago mapas conceptuales de los temas de cada una de las materias para estudiar posteriormente.

En la realización de esquemas utilizo muchas palabras.

Para realizar los esquemas, copio frases de los apuntes, libros y otros materiales de curso.

Organización

Subrayo las ideas principales en el material de estudio.

\section{Pensamiento crítico}

Trato de aplicar las ideas de las lecturas en otras actividades de clase como exposiciones y discusiones de clases

\section{Metacognición}

Prefiero el material que me cause curiosidad, aunque se me dificulte entenderlo.

Los términos o palabras confusos los consulto en un diccionario o enciclopedia.

Cuando leo hago preguntas que me ayudan a enfocar mi lectura.

Prefiero tener material que realmente me rete para poder aprender nuevas cosas.

\section{Manejo de recursos}

Tengo computadora en mi lugar habitual de estudio.

Cuando estudio, tengo a mano toda la información y materiales necesarios.

\section{Buscar ayuda}

Le pregunto al profesor o a la profesora para clarificar los conceptos confusos.

\section{Motivación intrínseca}

Lo más importante para mí es mejorar mi promedio general, así que el objetivo en este curso es obtener una buena nota.

Lo más satisfactorio para mí, en esta carrera es tratar de comprender el contenido lo más profundo como sea posible.

\section{Motivación extrínseca}

Quiero hacerlo bien en esta carrera porque es importante demostrar mi capacidad a mi familia, amigos, empleadores y otros.

\section{Ansiedad}

Al salir de un examen compruebo con los apuntos las respuestas que he dado.

Cuando hago un examen pienso en las preguntas que dejé sin respuesta.

Cuando hago un examen pienso en las consecuencias si fallo.

Cuando hago un examen siento que mi corazón late rápido.

Cuando hago un examen, tengo una sensación de inquietud y malestar.

0,39

Cuando tengo un examen me pongo nervioso (a). 